\title{
The Effect of Propofol on the Success Rate of Transurethral Lithotripsy by Holmium Laser: A Randomized Clinical Trial
}

\author{
Houman Teymourian', Saleh Ghiasy ${ }^{2,3}$, Ali Tayebi-azar ${ }^{4}$, Mohammadreza Razzaghi ${ }^{2}$, Ghazal Sadri ${ }^{5}$, Anahita \\ Ansari Djafari ${ }^{2}$, Seyyed Ali Hojjati ${ }^{2}$, Mohammad Poury' ${ }^{6}$, Vahid Shahabi ${ }^{2}$, Babak Javanmard ${ }^{2 *}$
}

\begin{abstract}
'Department of Anesthesiology and Critical Care, Shohada-e-Tajrish Hospital, Shahid Beheshti University of Medical Sciences, Tehran, Iran

${ }^{2}$ Department of Urology, Shohada-e-Tajrish Hospital, Shahid Beheshti University Of Medical Sciences, Tehran, Iran ${ }^{3}$ Laser Application in Medical Sciences Research Center, Shohada-e-Tajrish Hospital, Shahid Beheshti University of Medical Sciences, Tehran, Iran

${ }^{4}$ Nephrology and Kidney transplant Research Center, Urmia University of Medical Sciences, Urmia, Iran ${ }^{5}$ Resident of Radiology, Department of Radiology, Iran University of Medical Sciences, Tehran, Iran ${ }^{6}$ Clinical Research Development Center, Shohadaye Tajrish Hospital, Shahid Beheshti University of Medical Sciences, Tehran, Iran
\end{abstract}

\section{*Correspondence to Babak Javanmard, Department of Urology, Shohadae-Tajrish Hospital, Shahid Beheshti \\ University of Medical Sciences, Tehran, Iran. \\ Email: \\ drbabakjavanmard@gmail.com Tel: +98 9126988600; \\ Telfax: +982122736386}

Published online June 21, 2020

\begin{abstract}
Introduction: Urinary stone disease is the third most common affliction of the urinary tract that has been associated with an increasing incidence. Over decades, great advances have been made in the minimally invasive treatment of urinary stones. Recently, transurethral lithotripsy (TUL) by holmium laser was introduced as a possible therapeutic option. This study evaluated the effect of propofol on the success rate of TUL by holmium laser.

Methods: A double-blind randomized controlled trial was conducted on 180 patients to investigate the effect of propofol on the success and complication rate of TUL by holmium laser. The enrolled patients were divided into two groups: the first group received sodium thiopental $(n=89)$ while the second group received propofol $(n=91)$. The two groups were compared in terms of the fluctuations of systolic blood pressure (SBP), diastolic blood pressure (DBP), heart rate (HR), operation time, future stone-free rate (SFR), stone migration, post-operative fever, and ureteral complications such as perforation and mucosal damage. Other developed complications were also recorded. After data gathering, statistical analysis was performed with SPSS version 21.

Results: the patients' data such as age, sex, stone diameter, stone laterality, duration of stone impaction, primary SBP, DBP and HR were not significantly different between the two groups $(P>0.05)$. TUL and anesthesia duration, first-minute and fifth-minute SBP and DBP, and also changes of HR were significantly lower in the propofol group compared with the sodium thiopental group $(P<0.001)$. Moreover, SFR of TUL was more evident in the propofol group. Ureteral mucosal damage was significantly less in the propofol group.

Conclusion: Propofol was associated with a higher reduction in SBP and DBP, decreased duration of TUL, fewer fluctuations in HR, and an increased success rate of stone removal by TUL with holmium laser.

Keywords: Holmium laser; Propofol; Transurethral lithotripsy; Urolithiasis.
\end{abstract}

\section{Introduction}

Urinary stones are the third most prevalent disease of the urinary tract. ${ }^{1}$ The frequency of this condition varies in different regions with a prevalence rate of $1 \%-5 \%$ in Asia, $5 \%-9 \%$ in Europe and 3\% in Northern America, and it is growing by approximately $0.5 \%$ per year in the normal population of the United States of America and Europe. ${ }^{2}$

Currently, treatment protocols mainly focus on the prevention of new kidney stone formation and also inhibiting the enlargement of existing stones. ${ }^{3}$
The stone site has a major impact on the symptoms of acute renal colic. ${ }^{4}$ Over decades, great advances have been made in the minimally invasive treatment of ureteral stones. ${ }^{5-7}$ Recent therapeutic options include transurethral lithotripsy (TUL) by holmium laser, extracorporeal shock wave lithotripsy (ESWL) for proximal ureteral stones, and laparoscopic lithotripsy. ${ }^{5,8,9}$

With advances in laser technology, a wide range of laser settings and techniques that help in stone removal are available to surgeons. ${ }^{10-12}$ 
Stone removal with ureteroscope is most effective in treating lower ureteral stones. ${ }^{4}$ In the setting of acute ureteral colic, ureteral spasms make the passage of the guide wire of the ureteroscope difficult. ${ }^{5}$ However, applying local lidocaine gel, aminophylline 5\%, or intravenous Buscopan $®$ can reduce ureteral spasms and increase the rate of successful ureteroscopy. ${ }^{13}$

Propofol is a hypnotic drug from the alkylphenol group used for anesthesia and sedation during intensive care and surgery. ${ }^{14}$ In recent years, the use of propofol without muscle relaxants during laryngoscopy, intubation, and insertion of the laryngeal mask has been widely studied, showing that applying propofol without muscle relaxants induces smooth muscle relaxation during procedures. ${ }^{15}$

Based on this finding, the effect of propofol on decreasing muscle tone was approved, but the effect of this drug on ureteral spasm, tissue injury, and the success rate of TUL by holmium laser had not been studied. This study aimed to assess the effect of propofol on decreasing ureteral spasm, possibly leading to successful ureteroscopy and TUL by holmium laser.

\section{Methods}

Study Population

Participants with a mean age of $34.3 \pm 4.9$ and an established diagnosis of renal colic and a distal ureteral stone were enrolled in this study. All patients had been referred to
Shohada-e-Tajrish hospital between September 2016 and October 2017 for TUL operation. The exclusion criteria were: pregnancy, history of previous TUL, functional or anatomical renal disorders, difficult stenting, pulmonary disease, smoking, addiction, concurrent corticosteroid therapy, and allergy to propofol, egg, and soya.

\section{Study Protocol}

This study was designed as a double-blind clinical trial. After approval of our institutional review board, 180 patients were included in this prospective study and they received individual codes. Using computer-based software, the patients were randomly divided into two groups (Figure 1). To ensure blinding, the following method was used: an anesthesia technician loaded needles with a certain volume of each drug and labeled the needles with A or B. Then, according to a random number table, the needles were delivered to the blinded anesthesiologist. Furthermore, the surgeon and his/her assistant were not aware of the type of hypnotic drug used during TUL by holmium laser.

First, $1.5 \mathrm{mg} / \mathrm{kg}$ lidocaine, $2 \mu \mathrm{g} / \mathrm{kg}$ fentanyl and $0 / 02$ $\mathrm{mg} / \mathrm{kg}$ midazolam were administered to all patients to induce sedation and then $2.5 \mathrm{mg} / \mathrm{kg}$ propofol was prescribed for group one (propofol group) and $5 \mathrm{mg} / \mathrm{kg}$ sodium thiopental was prescribed for group two (sodium thiopental group). An appropriate laryngeal airway was

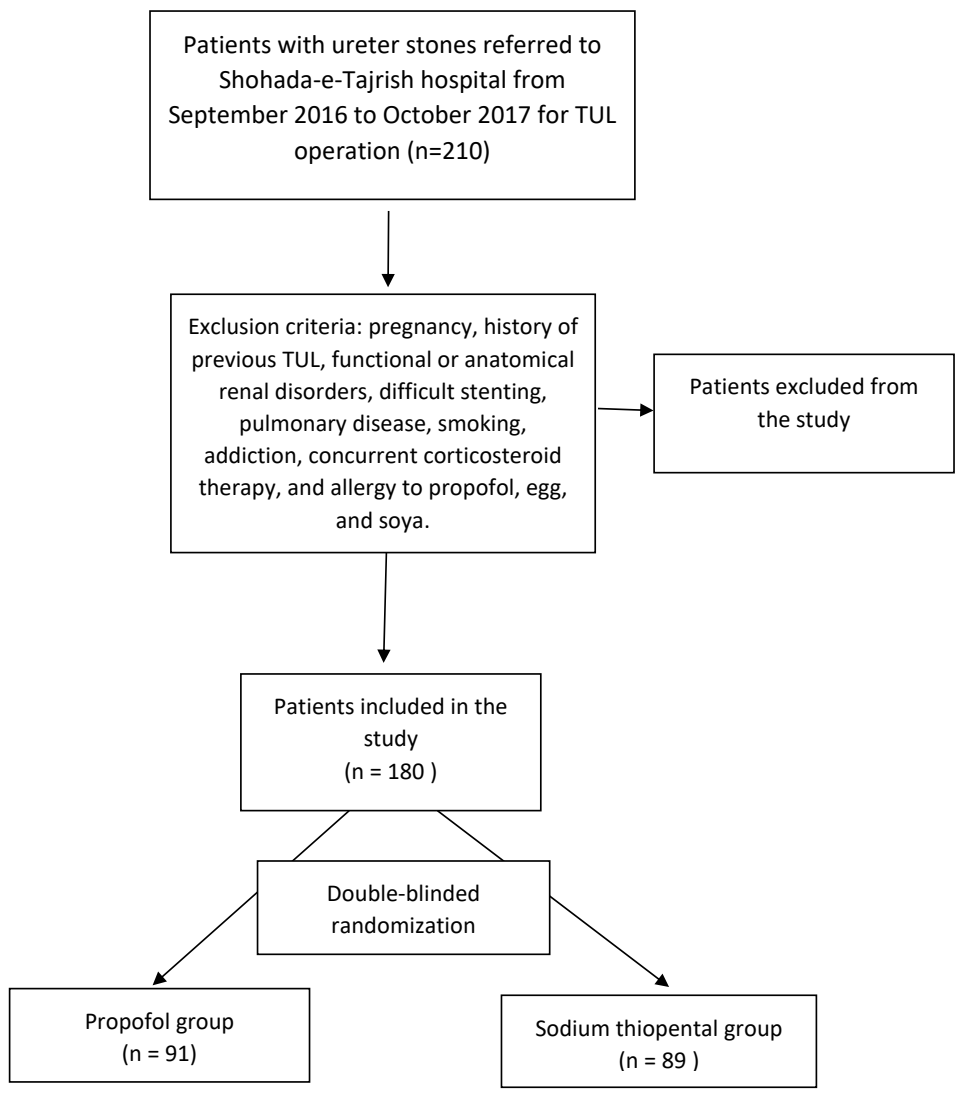

Figure 1. Study Protocol. 
inserted for both groups and inhalatory $\mathrm{O}_{2}, \mathrm{~N}_{2} \mathrm{O} 50 \%(3$ $\mathrm{L} / \mathrm{min}$ ), and 1\%-2\% MAC sevoflurane were administered for all patients. Ureteroscopy was performed with a $9.5 \mathrm{~F}$ semi-rigid ureteroscope in the lithotomy position (Wolf Inc., Germany). The Ho:YAG laser (manufactured in Iran) was used for lithotripsy. After stone visualization, the laser fiber $(500 \mathrm{Mm})$ was crossed through the working channel of the ureteroscope, and then the laser was discharged. Laser lithotripsy continued until stone fragments became as small as sand particles. A single urologist performed ureteroscopy combined with the Ho: YAG laser for all patients.

\section{Study Objectives}

The outcome of this study was to compare two groups in regard to fluctuations in systolic blood pressure (SBP), diastolic blood pressure (DBP), heart rate (HR), operation time, future stone-free rate (SFR), stone migration, post-operative fever, and ureteral complications such as perforation and mucosal damage.

Statistical Analysis

Statistical Package for Social Sciences (SPSS) software version 21.0 (IBM corp., Chicago, IL, USA) was used for statistical analysis. Quantitative variables are presented as mean \pm standard deviation and categorical variables are presented as frequency and percentage. All nonparametric comparisons were performed by chi-Square tests. A $P$ value of less than 0.05 was considered statistically significant.

\section{Results}

Eighty-nine patients were enrolled in the sodium thiopental group and 91 were included in the propofol group. The analysis showed that the patients' age, sex, stone diameter, stone laterality, duration of stone impaction, and baseline SBP, DBP, and HR were not significantly different between the two groups $(P>0.05)$ (Tables 1 and 2$)$. The mean duration of TUL and anesthesia was significantly less in the propofol group than the sodium thiopental group $(P<0.001)$. The evaluation of hemodynamic changes showed that first-minute and fifth-minute SBP, DBP, and HR fluctuation were significantly less in the propofol group than the sodium thiopental group $(P<0.001)$ (Table 2$)$.

Our results showed that immediate SFR was higher

Table 1. Demographic and Clinical Characteristics of Subjects

\begin{tabular}{|c|c|c|c|}
\hline Variables & Sodium Thiopental Group $(n=89)$ & Propofol Group $(\mathrm{n}=91)$ & $P$ Value \\
\hline Mean age $\pm S D$, (year) & $47.2 \pm 10.9$ & $46.3 \pm 10.4$ & $\geq 0.05$ \\
\hline Male, No. (\%) & $55(68.5)$ & $57.1(59)$ & $\geq 0.05$ \\
\hline Stone diameter $\pm \mathrm{SD},(\mathrm{mm})$ & $8.6 \pm 1.5$ & $8.7 \pm 1.6$ & $\geq 0.05$ \\
\hline Stone laterality (right), No. (\%) & $53(59.5)$ & $64(70.3)$ & $\geq 0.05$ \\
\hline \multicolumn{4}{|c|}{ Duration of stone impaction, No. (\%) } \\
\hline 1 month & $58(65.1)$ & $70(76.9)$ & $\geq 0.05$ \\
\hline Between 1 to 4 months & $14(15.7)$ & $14(15.3)$ & $\geq 0.05$ \\
\hline More than 4 months & $17(19.1)$ & $7(7.6)$ & $\geq 0.05$ \\
\hline
\end{tabular}

TUL, transurethral lithotripsy.

Table 2. Patients' Intraoperative and Postoperative Data

\begin{tabular}{|c|c|c|c|}
\hline Variables & Sodium Thiopental Group $(n=89)$ & Propofol Group $(\mathrm{n}=91)$ & $P$ value \\
\hline Mean anesthesia time $\pm S D$, (min) & $54.2 \pm 8.2$ & $43.5 \pm 8.7$ & $<0.001$ \\
\hline Mean TUL by holmium laser time $\pm \mathrm{SD}$, (min) & $43.2 \pm 8.2$ & $32.5 \pm 8.7$ & $<0.001$ \\
\hline Baseline SBP (mm Hg) & $138.7 \pm 12.9$ & $136.8 \pm 13.4$ & 0.3 \\
\hline Baseline DBP (mm Hg) & $77 \pm 7.6$ & $75.8 \pm 8.7$ & 0.3 \\
\hline Baseline HR (BPM) & $77.3 \pm 8.1$ & $77.6 \pm 7.7$ & 0.8 \\
\hline SBP (First minute) $(\mathrm{mm} \mathrm{Hg})$ & $128.6 \pm 12.9$ & $118.3 \pm 11.9$ & $<0.001$ \\
\hline DBP (First minute) $(\mathrm{mm} \mathrm{Hg})$ & $73.8 \pm 7.6$ & $65.3 \pm 10.9$ & $<0.001$ \\
\hline HR( First minute) (BPM) & $90.3 \pm 8.1$ & $67.8 \pm 9.9$ & $<0.001$ \\
\hline SBP (Fifth minute) $(\mathrm{mm} \mathrm{Hg})$ & $129.7 \pm 12.9$ & $117.1 \pm 11.9$ & $<0.001$ \\
\hline DBP (Fifth minute) (mm Hg) & $73.7 \pm 7.6$ & $62.3 \pm 10.9$ & $<0.001$ \\
\hline HR (Fifth minute) (BPM) & $83 \pm 8.1$ & $67.5 \pm 9.9$ & $<0.001$ \\
\hline Mean hospital stay \pm SD, (h) & $26.3 \pm 6.6$ & $25.7 \pm 6.2$ & 0.5 \\
\hline Immediate stone-free status, \% & 75 & 85 & 0.06 \\
\hline Stone migration, $\%$ & 11 & 8 & 0.4 \\
\hline Ureteral perforation, $\%$ & 4 & 2 & 0.4 \\
\hline Post-operative fever, \% & 6 & 8 & 0.7 \\
\hline Mucosal damage, $\%$ & 19 & 9 & 0.04 \\
\hline
\end{tabular}

SBP: systolic blood pressure, DBP: diastolic blood pressure, HR: heart rate. 
in the propofol group in comparison with the sodium thiopental group $(P=0.06)$, although this was not statistically significant. Furthermore, mucosal damage occurred significantly more in the sodium thiopental group compared with the propofol group $(P=0.04)$.

\section{Discussion}

Endoscopic treatment is most commonly used to manage obstructive and/or large stones. The challenges of intra-corporeal lithotripsy include problems associated with the endoscopic procedure (i.e. potential trauma to the urinary tract) and specific problems created by incomplete stone fragmentation and inadequate fragment elimination. ${ }^{16}$ Surgeons' experience is a determinant factor in the success rate of removing ureteric calculi with ureteroscopic holmium laser lithotripsy and also the development of complications. Operations performed by experienced surgeons are associated with fewer complications and higher success rates. Factors such as patients' sex, age, stone size, and location have not been shown to be significantly related to complications or success rates. ${ }^{17}$

Up to now, traditional anesthetic drugs and modalities have been used for the dilatation of the ureter and the facilitation of stone removal. Investigations are ongoing to seek an alternative drug which has the least respiratory and cardiovascular complications and also induces significant muscle relaxation of the ureteral wall and effective anesthesia. Thus, finding the best way to administer endoscopic treatment of ureteral stones with minor complications is currently a major challenge. The aim of this study was to assess the effect of propofol on the complication and success rate of TUL by holmium laser as compared with sodium thiopental.

In this study, the average decrease in SBP and DBP was more in the propofol group than the sodium thiopental group. However, the mean change in HR was less in the propofol group. In previous studies, ${ }^{16,18}$ it has been shown that sodium thiopental can cause a dose-dependent decrease in arterial blood pressure (BP), stroke volume, and cardiac output. These effects of sodium thiopental are due to the attenuation of myocardial muscles and increased venous return and less effect on vascular resistance. ${ }^{19}$ On the other hand, it has been reported that during anesthesia with propofol, decreased vascular resistance and vasodilation lead to decreased blood pressure, which is concordant with the results of our study. ${ }^{20}$ In general, direct negative inotropic effects of propofol are more than other anesthetic agents. ${ }^{21}$ The reports of previous studies show that propofol is associated with a stable hemodynamic effect and propofol-related decrease in BP and HR is dose-dependent, which is consistent with our findings. ${ }^{22}$

It has been proposed that propofol inhibits calcium entrance from receptor-operated calcium channels
(ROCCs) and voltage-operated calcium channels (VOCCs). In 2014, Zhong et $\mathrm{al}^{18}$ designed a study on the pulmonary vessel of a mouse and demonstrated that propofol affects both receptor-dependent and non-receptor-dependent pathways of smooth muscle contraction. Thus far, no study has investigated the effect of propofol on smooth muscle contraction of the ureteral wall. Based on previous evidence regarding the established effect of propofol on muscle relaxation, we decided to conduct a study to investigate the relaxation effect of propofol on the ureteral wall during the TUL process. Based on the results of our study, such as the decreased duration of TUL by holmium laser (easy and fast removal of stone), the higher success rate of TUL by holmium laser and less need for conversion into ESWL after TUL, we concluded that propofol might have a possible effect on decreasing the tonicity of the ureteral wall. However, we recommend further studies to confirm this hypothesis.

TUL is associated with disadvantages such as ureteral mucosal damage, urinary tract infection, sepsis, hematuria, post-operative increase in $\mathrm{BP}$, and prolonged operative time. The present study showed that the duration of TUL by holmium laser was significantly less in the propofol group. Moreover, a higher SFR was observed in the propofol group compared with the sodium thiopental group. In addition, ureteral mucosal damage occurred significantly less in the propofol group. Previous studies ${ }^{8,18}$ have reported that propofol decreases the tonicity of upper respiratory tract muscles. Moreover, propofol affects calcium exchange in vascular smooth muscle cells and also induces endothelial nitric oxide release, promoting decreased muscle tone of systemic vessels and subsequently, vasodilation and decrease in $\mathrm{BP}^{23}$ Thus, this can justify why the duration of TUL is less in the propofol group.

In our study, three patients developed UTI after surgery, treated with antibiotics. Also, hematuria occurred in two patients, in which the patients were treated with hydration and diuresis. The hematuria after TUL for these patients, treated and cured by serum therapy (hydration) and did not continue more than 1 day. In the patient who developed sepsis, successful treatment was achieved by administering antibiotics. Overall, using propofol during TUL by holmium laser increases the feasibility of this procedure as well as being associated with fewer complications.

\section{Limitations of the Study}

This study had some limitations. First, this was a singlecenter study. Second, the patients were not controlled in terms of such past medical history as cardiovascular disease (HTN, arrhythmia, etc). Third, the sample size was relatively small. Thus, we recommend larger multicentral studies to validate the results of this study. 


\section{Conclusion}

Based on the results of this study, applying propofol is associated with the higher success rate of TUL by holmium laser as well as fewer complications.

\section{Ethical Considerations}

This study was approved by the ethical committee of Shahid Beheshti University of Medical Sciences (ethics code: IR.SBMU.RETECH.REC.1397.865). The identity of all participants was kept confidential and all personal information was de-identified in the study. After explaining the risks and benefits of this therapeutic method, written informed consent was obtained from all patients. Additionally, this RCT study has been registered at the Iranian Registry of Clinical Trials website (identifier: IRCT20180606039987N1).

\section{Conflict of Interests}

The authors declare no conflict of interest.

\section{Acknowledgements}

The authors are thankful for the assistance of operative room staff due to their invaluable help throughout this study. Also, we thank the anesthesia technicians and anesthesia physician residents who helped the authors with data collection.

\section{References}

1. Pfau A, Knauf F. Update on nephrolithiasis: core curriculum 2016. Am J Kidney Dis. 2016;68(6):973-85. doi: 10.1053/j. ajkd.2016.05.016.

2. Shoag J, Tasian GE, Goldfarb DS, Eisner BH. The new epidemiology of nephrolithiasis. Adv Chronic Kidney Dis. 2015;22(4):273-8. doi: 10.1053/j.ackd.2015.04.004.

3. Anatol T, Pinto Pereira L, Simeon D, Sawh L. Risk factors for urinary tract calculi in Trinidad. Trop Med Int Health. 2003;8(4):348-53. doi: 10.1046/j.1365-3156.2003.01009.x.

4. Soomro HU, Hammad Ather M, Salam B. Comparison of ureteric stone size, on bone window versus standard softtissue window settings, on multi-detector non-contrast computed tomography. Arab J Urol. 2016;14(3):198-202. doi: 10.1016/j.aju.2016.06.006.

5. Shafi H, Moazzami B, Pourghasem M, Kasaeian A. An overview of treatment options for urinary stones. Caspian J Inter Med. 2016;7(1):1-6.

6. Portis AJ, Sundaram CP. Diagnosis and initial management of kidney stones. Am Fam Physician. 2001;63(7):1329-38.

7. Fallah Karkan M, Ghiasy S, Ranjbar A, Javanmard B. Evaluation of $200 \mathrm{Mm}, 365 \mathrm{Mm}$ and $500 \mathrm{Mm}$ fibers of Ho: YAG laser in transurethral lithotripsy of ureteral: a randomize control trial. J Lasers Med Sci. 2018;9(1):69-72. doi: $10.15171 /$ jlms.2018.14.

8. Moosanejad N, Firouzian A, Hashemi SA, Bahari M, Fazli M. Comparison of totally tubeless percutaneous nephrolithotomy and standard percutaneous nephrolithotomy for kidney stones: a randomized, clinical trial. Braz J Med Biol Res. 2016;49(4):e4878. doi: 10.1590/1414-431X20154878.

9. Pearle MS, Roehrborn CG, Pak CY. Meta-analysis of randomized trials for medical prevention of calcium oxalate nephrolithiasis. J Endourol. 1999;13(9):679-85. doi: 10.1089/ end.1999.13.679.

10. Aldoukhi AH, Roberts WW, Hall TL, Ghani KR. Holmium laser lithotripsy in the new stone age: dust or bust? Front Surg. 2017;4:57. doi: 10.3389/fsurg.2017.00057.

11. Fallah karkan M, Razzaghi MR, Karami H, Ghiasy S, Tayyebiazar A, Javanmard B. Experience of 138 transurethral urethrotomy with Holmium:YAG laser. J Lasers Med Sci. 2019;10(2):104-7. doi: 10.15171/jlms.2019.17.

12. Allameh F, Razzaghi M, Fallah-Karkan M, Hosseini B, Tayyebi Azar A, Ranjbar A, et al. Comparison of stone retrieval basket, stone cone and Holmium Laser: Which one is better in retropulsion and stone-free status for patients with upper ureteral calculi? J Lasers Med Sci. 2019;10(3):17984. doi: 10.15171/jlms.2019.28.

13. Barzegarnezhad A, Firouzian A, Emadi SA, Mousanejad $\mathrm{N}$, Bakhshali R. The effects of local administration of aminophylline on transureteral lithotripsy. Adv Urol. 2012;2012:727843. doi: 10.1155/2012/727843.

14. Marinella MA. Propofol for sedation in the intensive care unit: essentials for the clinician. Respir Med. 1997;91(9):50510. doi: 10.1016/s0954-6111(97)90082-2.

15. Gupta A, Kaur R, Malhotra R, Kale S. Comparative evaluation of different doses of propofol preceded by fentanyl on intubating conditions and pressor response during tracheal intubation without muscle relaxants. Paediatr Anaesth 2006;16(4):399-405. doi: 10.1111/j.1460-9592.2005.01783.x.

16. Scotland KB, Kroczak T, Pace KT, Chew BH. Stone technology: Intracorporeal lithotripters. World $\mathrm{J}$ Urol. 2017;35(9):1347-51. doi: 10.1007/s00345-017-2057-x.

17. Leijte JA, Oddens JR, Lock TM. Holmium laser lithotripsy for ureteral calculi: predictive factors for complications and success. J Endourol. 2008;22(2):257-60. doi: 10.1089/ end.2007.0299.

18. Chen X, Zhang XL, Liu L, Chen Y, Piao M, Zhang FJ, et al. Gas chromatograph-surface acoustic wave for quick realtime assessment of blood/exhaled gas ratio of propofol in humans. Br J Anaesth. 2014;113(5):807-14. doi: 10.1093/bja/ aeu193.

19. Ilkiw JE, Haskins SC, Patz JD. Cardiovascular and respiratory effects of thiopental administration in hypovolemic dogs. Am J Vet Res. 1991;52(4):576-80.

20. de Wit F, van Vliet AL, de Wilde RB, Jansen JR, Vuyk J, Aarts LP, et al. The effect of propofol on haemodynamics: cardiac output, venous return, mean systemic filling pressure, and vascular resistances. Br J Anaesth. 2016;116(6):784-9. doi: 10.1093/bja/aew126.

21. Gelissen HP, Epema AH, Henning RH, Krijnen HJ, Hennis PJ, den Hertog A. Inotropic effects of propofol, thiopental, midazolam, etomidate, and ketamine on isolated human atrial muscle. Anesthesiology. 1996;84(2):397-403. doi: 10.1097/00000542-199602000-00019.

22. Shah SB, Chowdhury I, Bhargava AK, Sabbharwal B. Comparison of hemodynamic effects of intravenous etomidate versus propofol during induction and intubation using entropy guided hypnosis levels. $J$ Anaesthesiol Clin Pharmacol. 2015;31(2):180-5. doi: 10.4103/09709185.155145.

23. Kassam SI, Lu C, Buckley N, Lee RM. The mechanisms of propofol-induced vascular relaxation and modulation by perivascular adipose tissue and endothelium. Anesth Analg. 2011;112(6):1339-45. doi: 10.1213/ANE.0b013e318215e094. 